\title{
VOLKSKUNDE UND URGESCHICHTE IN DEN SPIELEN
}

\author{
Béla GUNDA† \\ Lehrstuhl für Volkskunde Kossuth Lajos Universität \\ H-4010 Debrecen, Postfach 16, Ungarn
}

Alice Bertha GommE hat in ihrer klassischen Arbeit über die englischen, schottischen und irischen Spiele erörtert, daß in den Spielen Bräuche verbergen und sich in ihnen z. B. die Erinnerung an den Baum- und Steinkult, die Verehrung von Sonne, Mond und Wasser, an das Menschenopfer usw. finden lasse (GOMME 1894, 1898: I. 107, 347, II. 474, 503). Offensichtlich aufgrund von GoMME gelangte auch Géza RÓHEIM zu dem Ergebnis, daß sich in einem der ungarischen Kinderreime die Erinnerung an schamanische Heilungen verbirgt. Er fügt hinzu: „Das Heilen mit großem Lärm, Pfeife, Trommel und Rohrgeige zeigt den weltweit verbreiteten Typ des schamanenartigen Heilens, das Trommeln ist vielleicht das Überbleibsel des Vertreibens des Krankheitsgeistes“ (RÓHEIM 1912: 360-362; RÓHEIM 1925: 11-12). RÓHEIM beruft sich auf folgenden Kinderreim:

\section{Weihe, Habicht, was ist mit deinem Fuß? \\ Moskowiter-(Türken-)kind hat ihn verletzt, Ungarkind hat ihn geheilt, mit Pfeife, Trommel, Rohrgeige ...}

Ich möchte an einem noch anschaulicheren Beispiel belegen, daß sich in den Spielreimen ungarischer Kinder die Erinnerung an uralte Zaubereien entdecken läßt. Unter den Biharer Kindesprüchen von József FARAGÓ und Imre FÁBIÁN findet sich folgender (FARAGÓ-FÁBIÁN 1982):

\author{
Am Dorfende ist ein Mädchen, \\ Hat nach Landbutter Verlangen, \\ Gerinne, gerinne, \\ Klumpe so zusammen \\ Wie Juliskas Kopf. (Pósalaka)
}

Dieser Kinderreim ist eine Variante des auch auf ungarischem Sprachgebiet weitbekannten Butterzauberliedes (GUNDA 1983a: 34-39). Die Variante von Tápé lautet (BÁLINT 1977: 154): 


\author{
Am Dorfende ein schwangeres Mädchen \\ hat nach Landbutter Verlangen, \\ O mein Gott, schenk sie ihr, \\ Sie stirbt gleich wegen ihr.
}

Bereits früher, in meiner zitierten Studie, bemühte ich mich nachzuweisen, daß die in diesen Butterzauberliedern erwähnte weibliche oder andere Person irgendein böser Geist, die Verkörperung, der Nachkomme, eine verwandelte Gestalt der Hexe ist, der als Versöhnung, als Opfer von der Butter gegeben werden muß.

Zweifellos hat sich in den ungarischen Kindergedichtchen, den Butterliedern ein uralter Brauch erhalten. Ähnliche Varianten sind in England aus dem 16. Jahrhundert bekannt. Die Folkloreliteratur kennt auch heutige schottische und englische Varianten. Die eine der schottischen (KITTREDGE 1929: 169) lautet:

\author{
Komm, Butter, komm, \\ Komm, Butter, komm, \\ Peter steht am Durchgang (auf der Straße) \\ Und wartet auf den bebutterten Kuchen, \\ Komm, Butter, komm.
}

Der auf das butterbestrichene Zopfgebäck wartende geheimnisvolle Peter ist niemand anderes als das in unserem Biharer Spruch nach Butter verlangende Mädchen oder das nach Landbutter Verlangen habende schwangere Mädchen, also eine Art Hexe. Das belegt unter anderem die irische Variante (DAVIDSON 1958: 31), in der es sich bereits um eine Hexe mit Zauberkraft, um eine alte Vettel handelt:

\author{
Du giftige kleine Hexe, \\ Die durch die Gemarkung \\ Und kleinen geschützten Täler saust, \\ Komm her, und du findest mich, \\ Tu Butter in dies Butterfaß.
}

Das Butterzauberlied, von dem noch zahllose Varianten aufgezeichnet wurden, ist in ganz Europa bekannt, und der böse Geist, die Hexe, kann das Buttern ebenso fördern, wie sie das Verklumpen der Butter verhindern kann, sie zaubert die Butter zu sich. Gegen beide Hexenarten wird der magische Kampf ausgefochten (GUNDA 1983a: 38), aber statt dessen Einzelheiten gehört hierhin einzig die Lehre, daß unsere Kindersprüche die Erinnerung an den Zauber bewahrten.

In allen Teilen des ungarischen Volksgebietes ist - mit unwesentlichen Varianten - der Kinderspruch bekannt, den das kleine Kind beim Wegwerfen des herausgefallenen Zahns spricht:

Maus, Maus, gib mir einen Eisenzahn, Ich geb' dir einen Knochenzahn! 
Dieser Text wurde aus Bihar (Ant usw.) zitiert. Häfig tritt auch irgendeine Handlung zu dem Spruch hinzu: Man wirft den herausgefallenen Zahn über das Hausdach (FARAGÓ-FÁBIÁN 1982: 253), die Mutter steckt den Zahn in ein Mauseloch (Füzérkajata), man wirft ihn oben auf den Ofen oder in den Kessel (GöNCZI 1914: 226), vergräbt ihn unter dem Regenabflußrohr (Aszaló usw.).

Als ich begann, mich in der höchst verstreuten, fast unüberblickbaren Folkloreliteratur über die Sprüche zu informieren, wandte ich mich brieflich an den vorzüglichen norwegischen Folkloristen Christiansen Th. REIDAR. In seinem Brief (21. April 1959) wünschte er mir eine glückliche „Jagd“ für die Aufarbeitung des Themas. ${ }^{1}$ Das Sammeln des fast über alle fünf Kontinente verteilten Zauberspruches bzw. bereits eher spielerischen Kinderreimes war tatsächlich eine Jagd, eine Pfadsuche von einer Spur zur anderen. Ich zitiere keine rumänischen, südslawischen, slowakischen, tschechischen und anderen nahen Beispiele, sondern lieber zwei sehr entfernte. Auf Rarotonga, einer Insel im Stillen Ozean, sagt das Kind nach einem herausgefallenen Zahn: Große Ratte, kleine Ratte! Hier ist mein alter Zahn, Ich bitte dich, gib mir einen neuen! (FRAZER 1951: 179). Bei den Cherokee-Indianern (Georgia, USA) läuft das Kind mit seinem herausgefallenen Milchzahn um die Hütte und wiederholt fünfmal: Biber! Tu einen neuen Zahn in meinen Kiefer! (MONNEY 1900: 266).

Meines Wissens erscheint der älteste diesbezügliche Text in J. GRIMMs klassischer deutscher Mythologie. 1787 erwähnt er von Pforzheim, daß der ausgefallene Zahn in ein Mauseloch gesteckt wird, mit dem Spruch: Maus, da du Holzzähne hast, gib mir einen aus Knochen (GRIMM 1835: III, 454).

Die einzelnen Fälle haben namhafte Folkloristen so oder so erklärt (M. P. Saintyves, P. Sébillot, A. Schillings, B. Sueiro, J. R. dos Santos Jr.), sie versuchten, den sich auf die Kontinente verbreitenden Brauch zusammenfassend verständlich zu machen, doch verliert man sich fast im Kreuz und Quer der Theorien. So soll nur J. G. FRAZER erwähnt werden, der ziemlich umständlich den der Maus hingeworfenen Zahn zu jenem Zweig der sympathischen Magie rechnet, den er Übertragungsmagie (contagious magic) nennt (FrAZER, J. G. 1965: 43). Seine Beispiele belegen aber diese Auffassung nicht wirklich. Klarer erklärt den Sinn dieses Brauches J. DE VRIES, nach dessen Meinung die Geister in der Welt der Lebenden als tierartige Wesen (Schlange, Vogel, Maus, Schmetterling) erscheinen. Der Zahn wird deshalb der Maus vorgeworfen, weil dieses Tierlein die sichtbare Gestalt des Geistes ist. Diese Gestalt wurde deshalb gewählt, weil die mit starken und spitzen Zähnen versehene Maus (Ratte, Biber) mit dem Wunsch, mit der Hoffnung verbunden war, daß auch das Kind solche starken und gesunden Zähne bekomme (DE VRIES 1930: 8889). Diese akzeptable Erklärung konnte ich mit der Meinung von J. V. NEGELEIN ergänzen, daß sich schon bei den uralten Indoeuropäern ein mausgestaltiger ,Mensch' (Geist) an der Feuerstelle aufhielt, der die Milchzähne der Kinder als Op-

\footnotetext{
${ }^{1}$ Die Details der Aussagen über diesen Zaubervers finden sich in einem umfangreicheren Manuskript von mir. Auch Éva PóCS veröffentlicht Butterzauber-Beschwörungen und Sprüche über den ersten Zahn, doch sind ihre Literaturkenntnisse sehr mangelhaft (PÓCS, É.: Magyar ráolvasások [Ungarische Beschwörungen], I-II. Budapest 1985-1986).
} 
fer verlangte (NEGELEIN 1900: 291-293). Je länger wir bei dieser Frage verweilen, desto mehr verwickelt sich das Knäuel, und ein Fall unterstreicht die Theorien, der nächste widerlegt sie. Offensichtlich verkörpert sich der Anreiz der Maus, der Wunsch nach ihren kräftigen Zähnchen in jenem von Plinius erwähnten Brauch, gegen Zahnschmerzen monatlich eine Maus zu verzehren (KELLER 1909-1913: I, 197).

In unseren Kindersprüchen haben sich jedenfalls uralte Vorstellungen, magische Handlungen und sich fast über die ganze Welt erstreckende ähnliche Gefühle und Wünsche bewahrt, und diese werden auch von den Texten in den Kinderbüchern gefördert (KRESZ 1954: 15), welche mit den Butterzaubersprüchen zusammen beweisen, daß aus den einstigen Zauberworten ein Spiel geworden ist.

Betrachten wir einige Spielgeräte!

Seit Jahren beobachte ich, wo Kinder aus Ruten ein schlittenförmiges Spielzeug bastelten oder basteln, den sog. bakszekér, ,Bockkarren', der auch kleinere Lasten aushält. Dieses Frühlingsspielzeug der Kinder taucht als bakcsacsa, bakcsicsa, bakcsicsán, baki, bakszán, berszán vom Szeklerland über das Kalotaszeg bis nach Göcsej auf. Meines Wissens ist es in der Großen Ungarischen Tiefebene (mit Ausnahme des Banats) nicht bekannt. Es taucht in Österreich auf, bei den Rumänen (in Marmarosch ebenso wie bei den Pindus-Aromanen) und in der Hand der bulgarischen wie der wallonischen Kinder (GUNDA 1970: 221; MNL [Ungarisches Ethnographisches Lexikon]; A. PALÁdI-KovÁCs: Bakszekér; s. noch UMTSz [Neues Ungarisches Dialektwörterbuch]; KRESZ 1959: 188; KUNT 1974: 119-124). Bei einem gründlicheren Studium der westeuropäischen Malerei des 15.-17. Jahrhunderts taucht zur nicht geringen Überraschung auf einem Bild (Maria mit dem Jesuskind) von Lucas Cranach d. Ä. (1472-1553) unser Bockkarren (WEBER-KELLERMANN 1979: 76) auf. Es ist kaum anzunehmen, daß es sich um zufällige Übereinstimmungen handelt. Der Zusammenbau des bakszekér ist ziemlich kompliziert, und es besteht kein Zweifel daran, daß schon in viel früherer als der Zeit von Lucas Cranach damit gespielt wurde.

Es ist allgemein bekannt, daß eine wichtige Quelle der Kinderspielzeugforschung das Gemälde von Pieter Brueghel d. Ä. (um 1525-1569) mit den spielenden Kindern (1560) ist, über das Jeanette HILLS eine vorzügliche Monographie schrieb (HILls 1957); grundlegend ist aber auch P. PORTMANNs Arbeit (PORTMANN 1962), abgesehen von verschiedenen Teilstudien. Wir können uns freuen, daß András LUKÁCSY von den Darstellungen des Gemäldes mit lehrreichen Erklärungen ein Bilderbuch für die Jugend geschaffen hat (LUKÁCSY 1981). Weniger bekannt ist allerdings, daß es auch ein Kinderspiele darstellendes Gemälde von Maerten van Cleve (1527-1581) gibt. Bruegel hat 80 Kinderspiele (spielende Kindergruppen) dargestellt, letzterer 29. Der Vergleich beider Bilder ist eine gesonderte Aufgabe. Ich verweise nur darauf, daß beide Gemälde große Wirkung auf die holländischen Verfertiger von Stichen im 17. Jahrhundert ausübten (s. z. B. Nicolaes de Bruyn, 1570-1656, aus der Genrebildserie der Stich über die 10jährigen), deren Arbeiten dann zu Quellen der Spieldarstellungen der holländischen Wandkacheln des 17.-18. Jahrhunderts wurden. Ein vorzügliches Bild von der Spielliebe der Holländer zeigt übrigens die auch ungarisch erschienene Arbeit von P. ZUMTHOR (ZUMTHOR 1985: 
175-183). Vor allem auf Kacheln vor 1835 sind spielende Kinder häufig. Mit den Kacheln bedeckte man die Wände innen und außen, sie schützten gegen Feuchtigkeit. Seltener wurde auch der Boden von Küche und Stube gefliest. Auf den Kacheln und natürlich auch den Bildern von Brueghel und van Cleve tauchen fast alle Kinderspiele auf, mit denen auch die ungarischen Kinder spielen - selbst der heute so populäre Federball. Die Künstler der Kacheldarstellungen verewigten die reichen Varianten des Kreiselspiels. Selbstverständlich ist auch dieses Spiel sehr viel älter. Mit einem handgetriebenen Kreisel wurde in Theben schon um 1250 v. Chr. gespielt. Der mit der Peitsche getriebene Kreisel ist - wie so vieles andere - eine chinesische Erfindung. Auf den holländischen Ofenkacheln tauchen auch die Spiele mit Spielregeln auf, wie das Spatzeckspiel, das Steinfangen, das auch der holländische Maler Willem van Mieris (1662-1747) verewigte. Reichhaltige Varianten finden sich vom Paradiesspiel (ickázás, ugróiskola, emberezés usw.). Über die spielgeschichtliche Bedeutung der Kacheln erschien eine vorzügliche Monographie von Jan PLUIS (Pluis 1979, zur Geschichte des Kreiselns s. FraZER, A. (1966: 22-26, 38), vgl. noch GUNDA (1981: 65-69).

Um zu meinem eigentlichen Thema zurückzukehren, muß ich eingestehen, daß ich kein anderes früheres ikonographisches Dokument über den Bockkarren als das Gemälde von Lucas Cranach kenne. Auch auf den erwähnten Kacheln gibt es keine Bockkarren-Darstellung. Das schließt allerdings nicht aus, daß die Kenntnisse anderer in dieser Hinsicht weiter reichen. Irgendwo kann eine solche Darstellung noch auftauchen. Lucas Cranach ist weit herumgekommen. 1508 weilte er in Flandern und reiste durch wallonisches Gebiet, und es ist nicht ausgeschlossen, daß er gerade dort dieses Kinderspielzeug gesehen hat, das die Wallonen kennen. Ich setze voraus, daß die Kinder auch anderswo in Westeuropa solche kleinen Rutenschlitten bauen.

Wir dürfen nicht ohne jede Bemerkung über die identischen ungarischen und westeuropäischen Kinderspiele hinweggehen. Ich akzeptiere das Prinzip des Kindergedankens. Aber im Falle der nach komplizierten Regeln verlaufenden Spiele, so auch des Bockkarrens, müssen wir den Gedanken erwägen, daß diese Spiele überwiegend durch die im Westen gewesenen, dort studierenden Pfarrer, Lehrer und Reisenden mit pädagogischer Ader zu uns gelangt sind. Zu den Quellen der Spiele sind auch die damaligen pädagogischen Bücher zu rechnen, wie etwa der Orbis pictus von Comenius. Da die Lehrer und Pfarrer ihre Arbeitsstelle wechselten, trugen sie zusammen mit den pädagogischen Zeitschriften und Büchern zur sprunghaften heimischen Verbreitung der Spielvarianten und -benennungen bei. Damit läßt sich das Vorkommen der Benennungen cigele, cigere, kótya des Stockschlagens, Frosch/Pinzkerspielens in der Tiefebene und im Szeklerland (MTSz [Ungarisches Dialektwörterbuch], UMTSz [Neues Ungarisches Dialektwörterbuch]) erklären. Auf die Kollegien als Spielquelle lenkt die Aufmerksamkeit, daß es unter den Spielregeln auch Ausdrücke aus dem Lateinischen gibt (LÁNYI: Bige) und ein zwischen 1783 und 1791 aufgezeichnetes Schülerlied (BAKOS 1957: 273, auch Mária KRESZ macht - nach Ferenc Koós - auf die Kollegiumsherkunft der Volksspiele aufmerksam; KRESZ 1948: 19) ebenfalls auf das Kollegium als Quelle verweist: 


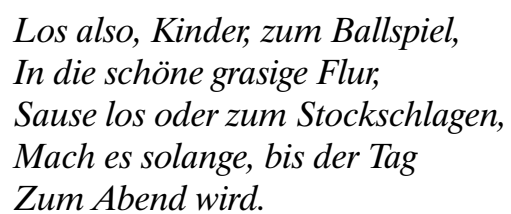

Die Verwendung und Verbreitung des Spielzeugs wurde durch den Handel gefördert. Bereits im 18. Jahrhundert gab es in Deutschland und Österreich eine weit entwickelte Spielzeugindustrie (Salzburg, Berchtesgaden, Nürnberg, Sonneberg, Marianberg, das Grödener Tal usw.). In den 1700er Jahren exportierte Oberammergau seine Spielzeuge nach St. Petersburg und Kopenhagen. Wir wissen auch, daß man mit Musterbüchern die Spielzeugherstellung förderte. Bei der Verbreitung der Spiele war das Schenken beteiligt. In Thüringen schenkte die Gräfin des Landes, Elisabeth, den armen Kindern Spielzeuge. Seit dem 19. Jahrhundert ist eine Möglichkeit für die Verbreitung der Spielzeuge der Brauch der Weihnachtsgeschenke.

Unter den Geduldsspielen gibt es ein ziemlich schwer zu öffnendes Teufelsschloß, das in der Literatur unter dem Namen chinesischer Ring oder meleda bekannt ist. Sein Sinn ist, vom Griff eines dickeren Drahtes den mit Ringen versehenen Teil abzunehmen und wieder anzubringen. Üblicherweise sind 6-12 Ringe an dem Griff, und bei der Variante mit 10 Ringen müssen nach komplizierten Regeln mehr als 100 „Durchschlingungen“ oder „Schritte“ vollführt werden. Deshalb ist es unmöglich, das „Öffnen“, das Trennen des Teufelsschlosses zu beschreiben oder mündlich zu erklären.

T. SZENTIVÁNYI hat sich in zwei hervorragenden Studien mit dem chinesischen Ring befaßt (SZENTIVÁNYI 1982a: 712-715; SZENTIVÁNYI 1982b: 17-18). Aus seinen Studien zitiere ich, daß , in China das Spiel Gastabschreckung genannt wird. Die Herkunft kennen wir nicht. Nach einer einstigen chinesischen Legende erfand es der chinesische Heerführer Hung Ming (181-234 n. Chr.) und gab es, als er in den Krieg aufbrach, seiner Frau, damit es ihr den Trennungsschmerz lindere. Mehr als 1000 Jahre später gelangte das Spiel nach Europa. Seine erste schriftliche Spur findet sich im Werk De Subtilitate Rerum des italienischen Naturwissenschaftlers Girolamo Cardano von 1550 (SZENTIVÁNYI 1982b: 17). Cardano beschrieb es als meleda. Dem kann ich hinzufügen, daß Girolamo Cardano ein leidenschaftlicher Spieler war und auch die Betrügereien der Spieler gut kannte. Er schrieb ein Buch über die Glücksspiele (De ludo aleae ...), bereiste Dänemark und Schottland, so daß er vermutlich an der Verbreitung der meleda beteiligt war. Wir wissen, daß meleda 1693 in England bekannt wurde. Im Wörterbuch von J. und W. Grimm stoßen wir auf dieses Geduldsspiel unter dem Namen Zankeisen, der 1541 auch in einem Nürnberger Witzblatt auftaucht. Die Bedeutung des Ausdruckes „Sie ist ein rechtes Zankeisen“ ist „Zänkische Frau“ (GUNDA 1984: 33-36). Noch einmal zu SZENTIVÁNYIs Forschungen zurückkehrend: Er schrieb, die mathematische Lösung der Öffnung der chinesischen Ringe, eines solchen Teufelsschlosses, habe 1872 der Franzose Louis Gros errechnet (SZENTIVÁNYI 1982a: 715; SZENTIVÁNYI 1982b: 17). Die Studien SZENTIVÁNYIs sollte jeder Spielforscher beachten. 
Zur meleda will ich noch hinzufügen (vgl. GUNDA 1984: 33-36), daß in Ungarn dieses Geduldsspiel ziemlich häufig ist. Schon Zsigmond BÁTKY teilte es 1903 aus Szentes unter dem Namen ördögbiblia ,Teufelsbibel' mit (BÁTKY 1903: 44-45). Aber meines Wissens wird es in der ungarischen Literatur erstmals 1840 aus Heves unter dem Namen cigánymiatyánk ,Zigeuner-Vaterunser' erwähnt (MTSz [Ungarisches Dialektwörterbuch]). Zuletzt hatte ich ein Exemplar in Händen, das die Zigeunerschmiede von Dorf Kovácsvágás in der Hegyköz Gegend verfertigt hatten. In den Dörfern des Zempléner Gebirges, in Szabolcs-Szatmár verfertigen oder bewahren die Zigeunerschmiede noch heute solche Geduldsspiele, die sie auch unter den Namen ördöglakat ,Teufelsschloß’, ördögmiatyánk, Teufels-Vaterunser' kennen. Aus den Mitteilungen geht nicht immer hervor, ob es sich tatsächlich um den meleda-Typ handelt (z. B. UMTSz [Neues Ungarisches Dialektwörterbuch]: cigánymiatyánk).

Eine ganz ähnliche Form dieses Teufelsschlosses ist in Schweden bekannt (Dalarna, Värmland, Östergötland usw.). Bei den Schweden ist es ein Kinderspiel, mit dem sich aber auch die Erwachsenen vergnügen. Nach Friedrich Freiherr von der Trenck wird es Trenck-Schlo $\beta$ genannt, verbreiteter ist aber die Benennung SinclairFessel. Die Tradition besagt, daß die Erfindung Malcolm S. Sinclair (eine aus Frankreich stammende Grafenfamilie, deren Mitglieder im 16. Jh. nach Schweden einwanderten) zu verdanken sei. Malcolm Sinclair kam 1709 bei Poltawa in russische Gefangenschaft und kehrte erst 1722 nach Hause zurück. Er also habe dieses Geduldsspiel erfunden und nach seiner Heimkehr in Schweden verbreitet. Der schwedische Ethnologe Sigfrid SVENSSON schreibt, daß dieses Spiel interkontinental verbreitet sei. In geschmackvoller Ausführung ist es auch im Handel zu bekommen. Seiner Meinung nach kehrte Malcolm S. Sinclair nicht in die Heimat zurück, weil ihn die Russen ermordeten (SVENSSON 1957: 1207; GUNDA 1984: 33-36).

Bei den Finnen heißt das Teufelsschloß sibirisches Schloß, bei den Esten eesti vigur ,estnischer Kniff' (KALJUVEE 1964: 229). Erstere Benennung verweist auf östliche Herkunft. In China und Ostturkestan ist dieses Geduldsspiel sehr populär. Nach meinen eigenen Beobachtungen vergnügen sich die Bulgaren und Tataren in der Dobrudscha noch heute mit diesem Teufelsschloß. Die rumänischen Bauern in Bihar und Oltenien kaufen es bei Zigeunerschmieden, die wie unsere eigenen Zigeunerschmiede nichts davon wissen, daß dieses Spiel bei uns wie in den USA unter Patentschutz gestellt wurde, was offensichtlich absolut überflüssig war (GUNDA 1984: 33-36).

Das Spielzeug kam als Warenartikel schon sehr früh in den Handelsverkehr. Darauf verweist auch SVENSSON. Zu unserer Überraschung taucht unter den über 1100 Spielen im Katalog des Nürnberger Kaufhauses Bestelmeier 1803 auch die meleda, unser Teufelsschloß, auf (SZENTIVÁNYI 1982a: 712 veröffentlicht eine Katalogseite). Daraufhin können sich die Spielforscher den Kopf zerbrechen, falls sie in irgendeinem Dorf auf ein solches ördöglakat, cigánymiatyánk stoßen, woher dieses wohl dahin gelangt ist.

Von den zahllosen Varianten von Teufelsschlössern erwähne ich nur noch ein aus zwei mehr oder weniger herzförmigen Gebilden bestehendes Geduldsspiel aus dem einzigen Grund, weil es auch in den USA aufgetaucht ist. Dorthin gelangte es 
vermutlich mit Einwanderern. Auch bei uns und anderswo in Europa (bei Polen, Esten, Russen usw.) ist es ein beliebtes Spielzeug, aber weniger kniffelig als die meleda. Das Teufelsschloß gelangte auch in die moderne ungarische Literatur. Mit dem Titel Ördöglakat erschien 1982 ein Gedichtband von István Kovács. In einem Gedicht ähnlichen Titels schreibt der Dichter: ,wir können das Teufelsschloß der Geschichte nicht auseinandernehmen". Auf dem Buchumschlag ist ein aus zwei dicken Nägeln gebogenes Teufelsschloß zu sehen. Nach ähnlichen Prinzipien wie bei diesem läßt sich auch das Teufelsschloß auseinandernehmen, das aus dünnerem Draht besteht und mit dem sich die Szekler vergnügen (GUNDA 1983b: 86; GUNDA 1984: 33-36).

Auch in der Spielforschung spielen die Werke von L. RÜTIMEYER und Th. DELACHAUX, die sich mit den primitiven Schweizer Kinderspielen beschäftigen, eine herausragende Rolle. Sie erklären die in großen Gebieten erkennbaren Ähnlichkeiten zu Konvergenzerscheinungen, zu denen der hochgradige Konservativismus der Kinderspiele beiträgt. Das Kind bringt die Spiele nicht nur von sich aus zustande, sondern deren primitiven Formen vererben sich von einer Generation auf die nächste. Die ersten Spiele für die Kinder verfertigt der Vater auf den Almweiden (RÜTIMEYER 1924: 163-167; DelaChaUX 1914: 101-112; DelaChaUX 1923: 190192). Aufgrund der kindlichen Psyche, der technischen Fertigkeit, des Nachahmungstriebs, der identischen Umgebung, der kindlichen Anziehung zu Tieren und ähnlichen Beobachtungen kann zwischen den Kinderspielen der verschiedenen Völker und Gebiete meistens nur psychische Verwandtschaft vorliegen. Aber es gibt eben - wie auch aus den vorgetragenen Fällen hervorgeht - auch kompliziertes technisches Spielzeug, regelgeleitete Gesellschaftsspiele. Auf deren Geschichte kann auch eine der wichtigsten Wissenschaften unserer Zeit, die Psychologie, keine Antwort geben. Ein bedeutender Teil der Spiele hat nicht nur eine Beziehung zur Psyche, zum Instinkt, und bringt nicht nur unser Gemeinschaftswesen zum Ausdruck, sondern sie stehen auch in Beziehung zu anderen Arealen, historischen Prozessen und zur Vergangenheit. Auf jeden Fall ist aber G. SCHLÄGERs Mahnung zu beachten, der die Kinderspiele für das Magma des primitiven Schaffenstriebs des Menschen hielt (SCHLÄGER 1917: 106-108). Beim Studium der Spiele können wir nicht nur vom Elementargedanken, vom Völkergedanken, sondern auch vom Kindergedanken sprechen. Wir sollten auch nicht vergessen, daß das Spiel den Menschen wie das Tier kennzeichnet und nicht nur im Verstand wurzelt, in welchem Fall es sich nur auf die Welt der Menschen bezöge. Das Spiel ist an keinerlei Kulturstufe, keinerlei Weltanschauung gebunden - sagt J. HuIZINGA (HuIZINGA 1956: 9-10). Am überraschendsten für mich ist der ,spielerische' Flug einander jagender Schmetterlinge.

Im Rahmen dieser Gedanken ist es lehrreich, einige konkrete Fälle in der Nachfolge der Forschungen von RÜTIMEYER zu betrachten. Es fällt auf, daß die holzgeschnitzten Kühe des Schweizer Evolena-Typs mit den Darstellungen des vor den Pflug gespannten Rindes auf den Zeichnungen in der Col di Tenda-Höhle (Neolithikum, Bronzezeit) übereinstimmen. Treffend wurde über diese urzeitlichen Rinder geschrieben, daß sie eher Käfern als Rindern ähneln (RÜTIMEYER 1924: 192). Natürlich schaffen nicht nur die Leute von Col di Tenda und die Schweizer Kinder 
ähnliche Formen, sondern z. B. auch die estnischen Jugendlichen, deren Kuhschnitzereien sich in nichts von den Kühen der Feldbauer von Col di Tenda und der Schweizer Kinder unterscheiden (KALJUveE 1964: 212, I. Abb. 2-3). Möglicherweise ist es wissenschaftlich gewagt, aus den Holzkühen auf Tierrassen rückzuschließen. Dennoch sage ich, daß die Spielkühe in der Schweiz (z. B. im Bündneroberland) teils von Hölzchen mit stellenweise entfernter Rinde, also mit Flecken, dargestellt werden (bunte Kühe), teils aus unabgerindeten Hölzchen (Simmental, braune Kühe) geschnitzt werden. Dies sind Kühe, wie sie in diesen Gegenden früher gezüchtet wurden (RÜTIMEYER 1924: 171). Es läßt sich beobachten, daß die Maiskolbenkühe und -ochsen in der ungarischen Tiefebene immer große Hörner haben, wie das graue ungarische Rind. Es gibt unter ihnen keine mit kurzen, abwärts gebogenen Hörnern. Lehrreich ist vom Gebiet am Nyikó zu lesen, daß die Kinder bei ihren Spielochsen ,ihre eigenen Kühe und Ochsen mit gebogenen, gabelförmigen oder kranartigen Hörnern nachahmen" (JÓZSEF 1943: 14).

Es ist allgemein bekannt, daß die Kinder in Ungarn Kühe und Ochsen aus Maiskolben, Maisstengeln und Kartoffeln herstellen; seltener aus Kastanien - wo es solche gibt. Die ersteren Pflanzen sind in Europa aber postkolumbianisch. Was hat es vorher gegeben? In der Tiefebene entstand das Spielzeug wahrscheinlich aus Holzstückchen, wie in einem großen Teil der Schweiz. In Gebirgsgegenden paßt zur Herstellung solcher Tiere sehr gut der Fichtenzapfen. Im Szeklerland sammeln die Kinder Lärchenzapfen (fenyö́csutika) und treiben sie als Schafe, sie spielen bácsolkodás ,Hüten' (GAZDA 1980: 225). Aus Fichtenzapfen verfertigen auch die Schweizer Kinder ihr Spielzeug. Man denke an Gottfried Kellers Novelle „Ursula“ (erschienen in den „Züricher Novellen“ 1877), wo die Frauen Fichtenzapfen an langen Strippen durchs Zimmer ziehen, da sie den Kindern kein anderes Spielzeug geben können. ${ }^{2}$ Fichtenzapfenspielzeug ist in verschiedenen Gegenden der Schweiz bekannt, Zapfenkühe werden aber auch in Katalonien und Frankreich hergestellt. Andererseits spielt man im Juragebirge nicht mit Fichtenzapfen. Wo es keine Fichtenzapfen gibt (Südtessin), ersetzt man sie durch Maiskolben, Gurken und Kartoffeln (RÜTIMEYER 1924: 180-182).

Die Fichtenzapfenkühe weiden und wandern auf lehrreichen sprachlich-urgeschichtlichen Wiesen und Pfaden. Das Wort für die Kuh loba kommt im deutschen Sprachraum der Schweiz in verschiedenen Varianten vor, z. B. in Vorarlberg, es bedeutet aber auch Fichtenzapfen. In Savoyen und im Unter-Engadin kennt man nur die letztere Bedeutung dieser sehr alten, wahrscheinlich vorkeltischen Terminologie. Die ursprüngliche Bedeutung ,Kuh' dieses Wortes ist schon verblaßt, und es bedeutet nur den Fichtenzapfen, aus dem sich die Kinder ihre Kühe machen. In mehreren Gebieten der Deutschschweiz ist loba, lobe, lobi das Rufwort für Kuh und Kalb oder der Name einzelner Kühe. Ich beschäftige mich nicht mit den verschiedenen Varianten von loba, lobe, lobi und mit seinen wortgeographischen und etymologischen Fragen, erwähne aber, daß die Sekundärbedeutung des hierzugehö-

\footnotetext{
${ }^{2}$ Gottfried KeLLERs gesammelte Werke. Bd. IV, Züricher Novellen. Verlag von Philipp Reclam Jun. Leipzig 1921: 379. Die Arbeiten des Verfassers enthalten reiche volkskundliche Details.
} 
renden lova, löve in Oberitalien ,Maiskolben' ist, also darauf verweist, daß die Kinder mangels Fichtenzapfen ihre Spielzeugkühe aus Maiskolben formen. Mit gewissem Humor kann ich sagen, daß dank der Kinder eine uralte, vorkeltische Terminologie dort am Maisstengel hängt. Die Bedeutung ,Rind' bzw. auch ,Fichtenzapfen' anderer vorrömischer Wörter (puscha, buscha, bütsch; muya, mouguet, Meil usw.) hat bewahrt, daß die Namen der Fichtenzapfentierlein früher oder gleichzeitig die Kuh und das Kalb selbst bedeuteten (JUD 1911: 9-17; RÜTIMEYER 1924: 194-206, über loba samt Varianten ist MEYER-LÜBKE 1935: 412-413, 472 anderer Ansicht).

Was hier ausgeführt wurde, ist schwer zusammenzufassen, doch konnte vielleicht eine Vorstellung von den sich in den Spielen verbergenden ethnographischen Lehren vermittelt werden. Gestehen wir ein, daß unsere Kinderspiele unentdeckt und unübersichtlich sind, daß wir die in ihenen steckenden kulturgeschichtlichen und ethnographischen Werte nicht kennen. Ihre Geschichte ist zumeist von einem Schleier verdeckt. ${ }^{3}$ Früher habe ich versucht, mit einzelnen Schülern einige Kinderspiele aufzuarbeiten, indem wir sie in den europäischen Horizont stellten. Dessen einziges greifbares Ergebnis war Edit Haiders gehaltvolle Facharbeit über das Paradiesspiel (ickázás). - Was aber soll nun geschehen? Meiner Ansicht nach wäre, auch um die weiteren Arbeiten voranzubringen - sogar nach den inhaltsvollen Wortartikeln über Spiele im Ungarischen Ethnographischen Lexikon - ein Lexikon der ungarischen Spiele notwendig, die kulturgeschichtliche, sprachliche, ethnographische, archäologische und sonstige Aufarbeitung der Spiele. Natürlich müßten auch die modernsten Spiele und die pädagogischen, Volksbildungs-, gewerblichen und Handelsansprüche berücksichtigt werden. Ich weiß sehr wohl, daß die Herstellung, das Schreiben eines Lexikons keine dankbare Arbeit ist. ${ }^{4}$ Aber betrachten wir diese Aufgabe nicht als Arbeit, sondern als die bleibendste, beständigste und angenehmste menschliche Tätigkeit: als Spiel ...

\section{LITERATUR}

BAKOS, J.

1957: Mátyusföldi gyermekjátékok (Kinderspiele aus dem Mátyusföld). Budapest. BÁLINT, S.

1977: A szögedi nemzet (Die Szegediner Nation). A Móra Ferenc Múzeum Évkönyve 1976-1977, 2. BÁTKY, Zs. Teil. Szeged.

1903: Farkas Sándor nagyalföldi magyar néprajzi gyújteménye (Ungarische volkskundliche Sammlung aus der Großen Tiefebene von Sándor Farkas). Népr. Ért. IV. Budapest 33-45.

\footnotetext{
${ }^{3}$ Wir sollten jedoch nicht solche hervorragenden Zusammenfassungen wie die von Mária KRESZ (KRESZ 1948) vergessen. Die Autorin bietet nicht nur eine Zusammenfassung, sondern auch neue Aspekte. Es scheint, daß sie sich eher die funktionelle Spielforschung zu eigen macht (12), sie berichtet aber auch gehaltvoll über die Ergebnisse der ungarischen ,mythologisch-historischen' Spielforschung. Es wäre notwendig, diese Studie gemeinsam mit anderen von ihr erneut herauszugeben.

${ }^{4}$ Die Kritiker mögen ungerechtfertigterweise die gründlich erarbeiteten, ihnen fernstehenden Stichwörter vom Tisch wischen, besonders, wenn ihnen die ethnographische Anschauung und die Kenntnis der Perspektiven fremd sind (s. z. B. Agrártörténeti Szemle, XXVI, 1984: 563-571).
} 
DAVIDSON, T.

1958: Cattle-Milking Charms and Amulets. Gwerin, Vol. II. No. 1, 22-37.

DelachauX, Th.

1914: Jouets rustiques suisses. Schweizer Archiv für Volkskunde, Bd. 18. Basel 101-112.

1923: La répartition géographique des jouets primitifs en Suisse. Actes de la Société elvetique des sciences naturelles. Zermatt, IIe partie, 19-192.

FARAGÓ, J.-FÁBIÁN, I.

1982: Bihari gyermekmondókák (Kindersprüche aus Bihar). Bukarest.

FRAZER, A.

1966: A History of Toys, London.

FRAZER, J. G.

1951: The Golden Bough. Part I. The Magic Art and the Evolution of Kings. Vol. I. New York.

1965: Az Aranyág (Der goldene Zweig). Budapest.

GAZDA, K.

1980: Gyermekvilág Esztelneken (Die Welt der Kinder von Esztelnek). Bukarest.

GOMME, A. B.

1894, 1898: The Traditonal Games of England, Scotland and Ireland, Vol. I-II. London.

GÖNCZI, F.

1914: Göcsej s kapcsolatosan Hetés vidékének és népének összevontabb ismertetése (Eine zusammenfassendere Darstellung des Gebietes und Volkes von Göcsej und des anschließenden $\mathrm{He}-$

GRIMM, J. tés). Kaposvár.

1835: Deutsche Mythologie. Bd. III. Göttingen.

GUNDA, B.

1970: Részletek a Magyar Néprajzi Lexikonból (Details aus dem Ungarischen Ethnographischen Lexikon). Debreceni Déri Múzeum 1967. évi Évkönyve. Debrecen.

1981: Múvelódéstörténeti jegyzetek a magyar gyermekjátékokhoz (Kulturgeschichtliche Notizen zu den ungarischen Kinderspielen). Ethnographia XCII. Budapest, 65-69.

1983a: Hungarian Butter Rhymes. International Folklore Review. Vol. 3. London. 34-39.

1983b: Notes on the Occurrence of the „Wonder Puzzle“. Tennessee Folklore Society Bulletin, Vol. 49, No. 2. Murfreeboro, Tenn. 86

1984: Das Teufelsschloß. Volkskunst, 7. Jg. München, 33-36.

HILLS, J.

1957: Kinderspielzeug von Pieter Brueghel d. Ä., Wien.

HUIZINGA, J.

1956: Homo Ludens. Reinbek bei Hamburg.

JÓZSEF, D.

1943: A Nyikó menti gyermek magakészítette játékszerei (Das von Kindern selbstgefertigte SpielJUD, J. zeug am Nyikó). A Székely Nemzeti Múzeum kiadványai, Nr. 4. Kolozsvár.

1911: Dalla storia delle parole lombardo-ladine. Bulletin de dialectologie romane, Bd. 3. Bruxelles.

KALJUVEe, G.

1964: Eesti rahvapäraseid laste mänguasju (...). Etnograafiamuuseumi Aastaraamat, XIX. Tallin,

KELLER, O. 210-237.

1909-1913: Die antike Tierwelt, Bd. I-II. Leipzig.

KiTTREDGE, G. L.

1929: Witchcraft in Old and New England. Cambridge, Massachusetts.

KRESZ, M.

1948: A magyar gyermekjátékkutatás (Die ungarische Kinderspielforschung). Budapest.

1959: Játék a kalotaszegi Nyárszón (Das Spiel in Nyárszó in Kalotaszeg). Néprajzi Közlemények, Bd. IV, Nr. 4. Budapest. 172-173.

1954: Csip-csip-csóka. Népi mondókák (Volkssprüche). Budapest.

KUNT, E.

1974: A „Bakiszekér“ Pányokon (Der „Bockkarren“ in Pányok). A Miskolczi Hermann Ottó MúLÁNYI, Á. zeum Közleményei, 13. Miskolc. 119-124.

1971: Bige. Magyar Néprajzi Lexikon I. (Ungarisches Ethnographisches Lexikon) Budapest, 284. 
LUKÁCSY, A

1981: Gyermekjátékok, azaz idősebb Pieter Brueghel mester ... nyomán készült képeskönyv (Kinder-spiele, d. h. Bilderbuch nach ... von Meister Pieter Brueghel d. Ä.). Budapest.

MEYER-LÜBKE, W.

1935: Romanisches etymologisches Wörterbuch. Heidelberg.

MONNEY, J.

1900: Myths of the Cherokee. Nineteenth Annual Report of the Bureau of American Ethnology, Part I. Washington.

NEGELEIN, V. J.

1900: Der armenische Volksglaube. Globus, Bd. 78. Braunschweig 288-293.

PALÁDI-KOVÁCS, A.

1971: Bakszekér (,Bockkarren’). Magyar Néprajzi Lexikon I. (Ungarisches Ethnographisches Lexi-

PLUIS, J. kon) Budapest, 194-195.

1979: Kinderspelen op tegels (Kinderspiele auf Kacheln). Van Gorcum, Assen.

PoRTMANN, P.

1961: Die Kinderspiele: Pieter Brueghel d. Ä. Bern.

RÓHEIM, G.

1912: Sámánkodó gyógyítás nyoma egy gyermekversben (Spuren des schamanischen Heilens in einem Kinderreim). Ethnographia, XXIII. Budapest, 360-362.

1925: Magyar néphit és népszokások (Ungarischer Volksglaube und Volksbräuche). Budapest.

RÜTIMEYER, L.

1924: Ur-Ethnographie der Schweiz. Basel.

SCHLÄGER, G.

1917: Einige Grundfragen der Kinderspielforschung. Zeitschrift des Vereins für Volkskunde, Bd. 27. Berlin, 106-121, 199-212.

SVENSSON, S.

1957: Sinclairs bojor. Svensk upplagsbok, Bd. 25, Malmö, 1207.

SZENTIVÁNYI, T

1982a: Korunk logikai játékai (Die Logikspiele unserer Zeit). Élet és Tudomány. Budapest, 712-715.

1982b: Kínai karikák ... (Chinesische Ringe). És játék, Nr. 2. Budapest, 17-18.

DE VRIES, J.

1930: Le jet de la dent. Revue Anthropologique, Vol. 40. Paris, 87-89.

WEBER-KELLERMANN, I.

1979: Die Kindheit. Kleidung und Wohnen, Arbeit und Spiel. Insel Verlag. Frankfurt am Maim.

ZUMTHOR, P.

1985: Hollandia hétköznapjai Rembrandt korában (Hollands Alltage zur Zeit Rembrandts). Budapest. 